\title{
Factores de riesgo y protección del consumo de drogas entre estudiantes universitarios salvadoreños, año $2007 .{ }^{1}$
}

\author{
Drug Consumption Factors Of Risk And Protection \\ Among Salvadorian College Students, Year 2007.
}

\section{Resumen}

H l consumo de drogas es multicausal, se incluyen la disponibitidad y tráfico de drogas que se protifera en el país, depende en gran medida de la falta de prevención enfocada a fortalecer zalores, actitudes, conocimientos y habilidades desde el hogar, escuela y Estado. Se realizaron entrevistas a profundidad a expertos en el tema y por escrito a una muestra de 382 estudiantes, distribuidos estratificadamente en 8 Universidades. Según el análisis de indicadores estudiados se advierte que alrededor de una tercera parte de población, se encuentra en inminente riesgo: inseguridad individual, familiar y socioeconómica entre otras. La publicidad induce a la adopción de conductas inapropiadas y a consumo de drogas licitas e ilicitas y de todo tipo de vicios. Si a ello se le suma, que la Universidad prioniza la instrucción profesional y muy poco la educación inlegral; se genera una relación inversamente proporcional de factores de riesgo y protección: mientras se propicia el desgaste de factores de protección como la práctica de valores entre otros, se permite el aumento de factores de riesgo de todo tipo y con ello el consumo de sustancias psicoactioas. Ante el creciente riesgo, se adzierte que la respuesta de la Universidad, familia y Estado debe ser inmediata.

Palabras clave: Riesgos, prevención, drogodependencias entre universitarios.
E. Aydé Rivera de Parada Licenciada en Ciencias de la Educación.

Directora de Investigación Universidad Evangélica de El Salvador E-mail:aydeerivera@gmail.com 


\section{Introducción}

El consumo de alcohol y tabaco es una costumbre altamente común alrededor del mundo, por ello se constituve en uno de los principales factores de riesgo para los jóvenes y niños, seguidas de la amplia gama de otros tipos de drogas menos comunes y más peligrosos. El consumo de todo tipo de drogas tiene efectos de corto y largo plazo, desde cambios en el estado de ánimo, alteración de la capacidad de juicio hasta pérdida de apctito, cambio en ritmos de sueño, vigilia y daños pulmonares, cerebrales y de otros órganos, hasta producir la muerte.

En El Salvador el consumo de drogas ha sido un fenómeno cada vez más fuerte, en la última década ha afectado cada vez más a los niños y adolescentes, hasta el punto de conocer de distribución y consumo de drogas en centros de educación básica, con poco o nulo protagonismo de las Universidades en la prevención de éste.

Según la Organización Mundial de la Salud (OMS), droga es "toda sustancia que introducida al organismo por cualquier vía de administración, produce una alteración, de algún modo, del natural funcionamiento del sistema nervioso central del individuo y es, además, susceptible de crear dependencia, ya sea psicológica, física o ambas." La clasificación más general de drogas incluye: Alcohol, tabaco, marihuana, cocaina y crack, inhalantes, anfetaminas y metanfetaminas; tranquilizantes e hipnóticos; heroína, alucinógenos y éxtasis. Los tipos de drogas mencionados de acuerdo con su legalidad de uso se dividen en drogas lícitas e ilicitas, sin embargo el hecho de que algunas sean permitidas (ej. alcohol y tabaco) no niega las repercusiones en la salud.

\section{1.- Antecedentes del consumo de drogas en El Salvador}

Desde hace más de una década, se han creado los observatorios interamericanos, centroamericanos y nacional sobre drogas; en ellos se recogen datos que muestran la tendencia de proliferación del fenómeno; por lo que también los observatorios y organismos relacionados, incluyen programas de prevención y tratamiento en diferentes etapas de su evolución y en la particularidad de grupos afectados.

Un perfil de los pacientes atendidos por la Fundación Antidrogas de El Salvador (FUNDASALVA), elaborado en 1999, muestra la tendencia de incremento de consumo de drogas. La mayoría de pacientes atendidos eran hombres, jóvenes menores de 25 años, prevaleciendo estudiantes de bachillerato, de las capas media baja. Ei tabaco, la marihuana y la cocaína son las preferidas; tambièn se incluye el consumo de crack, el cual rápidamente está alcanzando una alta prevalencia; de cada 10 pacientes, 6 muestran un nivel severo de adicción. Es alarmante la detección de adicción a niveles severos. Según el informe de evaluación de progreso de control de drogas 2001-2002, hasta ese año El Salvador ha logrado un progreso sustancial en la ejecución de sus esfuerzos nacionales contra las drogas. La detección de muevas tendencias en el abuso de sustancias (éxtasis) y el aumento significativo en el tráfico ilicito de drogas hacia el país por tierra y por mar son motivo de especial atención por parte de las entidades encargadas del cumplimiento de la ley. Por lo tanto, parece que es necesario fortalecer la cooperación operativa fronteriza y regional.

Según el Consejo Nacional de Seguridad Pública y PNUD, 2004, citado por PNUD 2005 : "El consumo y el tráfico de alcohol y drogas constituyen importantes factores potenciadores de la violencia, en tanto tienen efectos directos en el comportamiento de las personas que los consumen, porque pueden motivar a los adictos a delinquir para conseguir el dinero necesario para su compra, y porque estimulan un contexto de violencia en torno a los sitios de compra y venta"

Según Rivas I. (2006), la población que más consume drogas sigue siendo la masculina, ya que según estudio realizado en instituciones de rehabilitación de El Salvador ${ }^{2}$, muestra la existencia de prevalencia en $86.1 \%$ para hombres y $13.9 \%$ para mujeres. Entre las causas más comunes del consumo están: satisfacer curiosidad, necesidad de aceptación al grupo, conseguir sensación de bienestar y tranquilidad; falta de comunicación familiar y falta de dedicación de los padres en su educación. Las drogas más consumidas son cigarrillos, alcohol, marihuana, cocaina y crack.

Actualmente, para darle seguimiento a este fenómeno se han desarrollado varios esfuerzos, entre ellos la creación de la Ley Reguladora de las actividades relativas a las Drogas que le anteceden leves similares; se creó una Comisión Salvadoreña Antidrogas (COSA) hoy Comisión Nacional Antidrogas (CNA) y se aprobó un Plan Nacional Antidrogas 2002-2008, el 22 de enero de 2002.

Fn el documento de aprobación del Plan Nacional Antidrogas 2002-2008, se refleja el incremento de la demanda a partir de 1998 que se ha experimentado un aumento en el consumo de crack, cocaína y drogas sintéticas como éxtasis. Esta última ha aparecido en discotecas en zonas de alta plusvalía.

A mediados del año 2000, con el apoyo de la Comisión Interamericana de Control y Abuso de Drogas (CICAD) y el Instituto Nacional sobre el abuso de

\footnotetext{
${ }^{2}$ El estudio se realizó en Fundación Desafio Juvenil Teen Chalenger, Fundación AIma, FUNDASAIVA y Hogares Crea. Según el decreto 153 de la Asamblea legislativa de la República de El Salvador, publicada en Diario Oficial el 7 de noviembre de 2003 y puesta en vigencia ese mismo año.

4 Dicho Plan fue aprobadu por la Asamblea Legislativa a solicitud de la COSA y se orienta la reducción de la oferta y demanda eon cuatro jes transversales de trabajo: con miras a la creación de un observatorio salvadoreño sobre drogas (investigación y estadisticas), cooperación internacional, legislación y capacitación.
} 
Drogas (NIDA) de los Estados Lnidos de América, se llevó a cabo una investigación sobre el abuso de drogas, que se realizó en Panamá, Centroamérica y República Dominicana, denominado "PACARDO", el cual se realizó en cada país para explorar el uso y consumo de alcohol, tabaco y drogas en la población escolar de 13 a 17 años de edad.

El estudio en El Salvador, tuvo una muestra de 1,615 escolares de colegios públicos y privados y según los resultados de dicha investigación:

\section{"El alcohol fue la droga que two la mayor oportunidad de uso por los escolares, donde más del tercio el $42.8 \%$ ha tenido la oportunidad de usar alcohol, seguido por el tabaco con un $30 \%$, la marihuana con un $8.7 \%$, es decix, uno de cada 12 alumnos ha tenido la oportunidad de usar esta sustancia" (COSA, 2000).}

Hay instituciones que colaboran en la tarea de prevención; pero sin el compromiso real y el esfuerzo compartido de todos los ámbitos, será imposible obtener logros significativos. Es necesario tener leyes para limitar la comercialización de las drogas, pero también es importante prevenir su consumo y un proceso de prevención debe comenzar por conocer la realidad. Los esfuerzos de prevención del uso de drogas deberían considerar la trilogía hogar, escuela y estado.

\section{2.-Tratamiento, prevención y programa preventivo.}

Según Becoña Iglesiaš (2002). El tratamiento es la atención directa a las personas que son víctimas del consumo regular, adicción o dependencia de sustancias psicoactivas; mientras que el término 'prevención' puede llegar a carecer de significado por ser muy general, el programa preventivo se pretende que sea específico en la medida que vaya orientado a objetivos concretos o grupos claramente delimitados, para tomar medidas más directas, hacer diseños más realistas y lo más importante e indispensable es evaluar el impacto de dicho programa.

"Más recientemente, y aplicado a la prevención de las drogodependencias, se ha introducido una distinción entre los siguientes tipos de prevención: universal, selectiva e indicada. Propuesta esta distinción inicialmente por Gordon (1987) y aceptada por el NIDA (Sloboda y David, 1997), ha sido rápidamente aceptada por los expertos y trabajadores en este tema (Gilchrist, 1995)" (Becoña Iglesias 2002).

\section{Prevención específica e inespecífica.}

Calafat en 1995 (citado por Becoña I.) distinguió la prevención específica de la inespecífica del siguiente modo: "Muy brevemente entendemos por prevención específica aquellas actuaciones que de una forma clara, concreta y explícita tratan de influir en el uso de drogas. Por contra, la inespecífica es la que trata de alterar los consumos indirectamente, a través de programas o actuaciones o ámbitos nada conectados en principio con el uso de drogas".

Por consiguiente los Lineamientos Hemisféricos de la CICAD (2005), adoptan los niveles de prevención universal, selectiva e indicada. En el nivel universal, las medidas de prevención se enfocan a fortalecer valores, actitudes, conocimientos y habilidades, mientras que la atención selectiva de prevención a través de programas, está dirigida a grupos o subgrupos de la población en mayor riesgo de consumo de drogas y el nivel de prevención indicada busca el tratamiento concreto a grupos o personas consumidoras.

En los niveles de prevención, la cvaluación diagnóstica debe hacerse en base a los factores de riesgo y prevención para saber en qué medida están presentes, si favorecen o no el consumo de drogas o el riesgo de cacr en el consumo.

\section{3.- Factores de riesgo y protección}

El conocimiento de factores de riesgo y protección para el consumo de drogas es importante para garantizar resultados de programas de prevención. Según Becoña I. 2002, "Se entiende por factor de riesgo -un atributo y/o característica individual, condición situacional y/o contexto ambiental que incrementa la probabilidad del uso y/o abuso de drogas (inicio) o una transición en el nivel de implicación con las mismas (mantenimiento)(Clayton, 1992, p. 15). Por factor de protección -un atributo o característica individual, condición situacional y/o contexto ambiental que inhibe, reduce o atenúa la probabilidad del uso y/o abuso de drogas - la transición en el nivel de implicación con las mismas- (Clayton, 1992, p. 16)".

Orte (1993), citado por Becoña, indica que hay toda una serie de factores que hacen dificil y compleja la comprensión de causales en el consumo de estas sustancias como el consumo independiente o la combinación de varias de ellas, que no se llega al consumo crónico de forma repentina, los daños dependen del estado fisico y de la consistencia de las drogas por lo que no se puede hablar de efectos universales de las drogas.

Factores de riesgo según Moncada (1997). Gitado por Becoña I. para ilustar como visualizan los factores de riesgo del abuso de sustancias spicoactivas.

1. Factores de riesgo ambientales;

2. La deprivación social;

3. La desorganización comunitaria;

4. La disponibilidad y accesibilidad de las sustancias;

5. La percepción social del riesgo;

6. La movilidad de la población;

7. Las normas y leyes de la comunidad: 
8. Factores de riesgo del individuo y sus relaciones con cl entorno;

9. Historia familiar de alcoholismo.

10. Pautas educativas (ej. relación vertical decente-estudiante, desconfianza);

11. Actitudes y modelos de conducta por parte de los padres;

12. Conflictos familiares;

13. Los valores;

14. La agresividad;

15. La busqueda de sensaciones;

16. Otros problemas de conducat;

17. Las actitudes hacia las drogas;

18. El fracaso escolar;

19. El grupo de iguales.

\section{Justificación del estudio}

Según estudio de prevalencia realizado por la CNA en el 2004, indica que la edad de inicio del consumo es entre los 11 y 12 años; al menos 4 de cada 10 jóvenes entre 13 y 17 años ha consumido alcohol o tabaco alguna vez en su vida; la prevalencia de vida para la marihuana es de $1.5 \%$ a diferencia del año 2001 que era de $0.1 \%$, lo que resulta alarmante. Debido a la amenaza de incremento de consumo de drogas; la CNA también plantea una iniciativa de prevención en preescolares de 4-6 años y a pesar de haber realizado la prueba piloto en una escuela del municipio de Apopa en el año 2005, aun no se ha continuado su implementación masiva, que podría ser una estrategia prometedora en estas edades; aunque sólo se podrian evaluar cambios sociales hasta después de algunas décadas.

Según el primer estudio de la población general realizado por CNA en 2006, un 4\% de la población en general ha consumido marihuana alguna vez en su vida y al menos 8 de 100 consideran ese consumo como nada o poco grave, en porcentajes entre 5 y $1.5 \%$ de personas consideran que el consumo frecuente de cigarrillos, tranquilizantes, estimulantes y bebidas alcohólicas es nada o poco grave; estos datos resultan preocupantes, pues ya se sabe que la exposición a este tipo de consumo o el simple hecho de modelaje familiar y social genera riesgo, pero para algunas personas es algo normal y no grave.

Como se ha ilustrado existe respaldo legal $\mathrm{e}$ iniciativas de pais bastante buenas, pero el problema aumenta a medida pasan los años y la necesidad de intervenir tempranamente es cada vez mayor. Los esfuerzos realizados han estado más enfocados al control de tráfico y tratamiento; para la prevención, el trabajo se ha enfocado a estudiantes de primaria y bachillerato.

En las universidades ha sido poco el esfuerzo y no hay estudios de esta naturaleza; sin embargo; se reconoce que es un lugar importante para prevenir y controlar, preparando jóvenes y grupos de docentes, para poder multiplicar los esfuerzos de promoción de estilos de vida saludables libre de drogas.

\section{Objetivo del estudio}

Diagnosticar factores de riesgo y protección del abuso de drogas entre jóvenes universitarios, para adoptar estrategias de intervención temprana en esta población.

\section{Metodología}

El estudio exploratorio se enfocó a la población de $36,797.00$ estudiantes de 8 universidades pertenecientes a la Asociación de Universidades Privadas de El Salvador AUPRIDES (según informe calificación MINED 2004); error muestral $0.05 \%$, muestra de 382 estudiantes, de éstos el $43 \%$ correspondió a hombres y el $56.5 \%$ a mujeres. La distribución estratificada por universidad se realizó en base al factor de proporción de 0.01038128 . La unidad de análisis son los estudiantes de comunidades universitarias, como grupos ubicados en los niveles de prevención universal y prevención selectiva, según clasificación de la CICAD; se incorporó un grupo de expertos como informantes clave. Se retomaron las variables e indicadores considerados en los Lineamientos Hemisféricos de la CICAD, aplicados a escuelas básicas, adaptando su contenido a la población universitaria.

\section{Instrumentos y técnicas.}

Se utilizaron 3 instrumentos de recolección de información (dos guias de entrevistas una para cntrevista a expertos, otra para entrevistas a docentes y conocedores de la materia y un cuestionario semi estructurado para estudiantes). Se realizaron entrevistas a expertos o informantes claves de diferentes instituciones relacionadas con prevención de uso indebido de drogas. Se realizaron 7 entrevistas individuales y grupales entre docentes y profesionales conocedores del tema, para recoger sus puntos de vista y analizar estrategias para prevención y atención.

\section{Datos generales}

De la muestra de estudiantes, 382, la mayoría son menores de 24 años $(68 \%)$; la mayoría vive en los municipios San Salvador 24\%, Soyapango $11 \%$ y Mejicanos. Trabaja y estudia un 39\%. Los responsables de su manutención y estudios son padre y madre $(43 \%)$; sólo madre $12 \%$, sólo padre $20 \%$, el mismo estudiante $28 \%$, tío/a $9 \%$, hermano/a $7.3 \%$. Al menos 3 de cada 10 estudiantes han interrumpido sus estudios universitarios por razones económicas y falta de tiempo disponible, por falta de madurez o aptitud para la carrera o por problemas de salud.

\section{Resultados}

Según la perspectiva de especialistas que trabajan con instituciones relacionadas con la prevención del consumo y tratamiento de personas 
adictas; se expone un resumen de factores de riesgo y protección o prevención ante el consumo de drogas lícitas e ilícitas. El consumo de drogas es un problema muy antiguo, pero cada vez se ha venido proliferando, en la medida que los intereses económicos están por encima de todo valor humano, se ha asociado a las modas juveniles, modelaje de familiares, artistas y personajes famosos promovidos por la publicidad, condición que se fortalece con los problemas sociales como la desintegración familiar, emigración, mayor desempleo, más personas desocupadas, éstas a su vez generan frustraciones, baja autoestima y otras limitaciones personales que predisponen al consumo de drogas, no sólo a los jóvenes sino también adultos y niños.

Factores individuales. Se logra percibir un deseo subyacente de superación personal y los jóvenes dejan ver una intensa lucha por lograrlo a costa de las situaciones personales, familiares y sociales que irrumpe su desarrollo. Los mismos jóvenes se valoran más por las incontables virtudes que poseen, que por sus aspectos negativos que quieren cambiar o mejorar; los aspectos que quieren cambiar son carácter, aspectos de personalidad y manera de ser, aspectos que parecen muy generales pero muestra el reconocimiento de la necesidad. El reconocer la necesidad de cambios es un indicador de sus deseos de superación.

Factores familiares y comunicación asertiva. La mayoría de jóvenes tiene familias nucleares, la mitad de jóvenes $(51.3 \%$ ) vive con padre y madre, sólo con madre $22 \%$, solo padre $2.4 \%$. Cuando tienen problemas al menos la mitad de los entrevistados recurre a la madre y 3 de cada 10 a su pareja o amigos, a diferencia de cuando necesitan dinero casi todos recurren a familiares; las razones anotadas es porque tienen confianza en esas personas.

La mayoría de los entrevistados expresan tener excelentes relaciones interpersonales en su familia. Estos resultados dan la esperanza de protección y prevención para que los jóvenes no caigan en situaciones lamentables; anotan aspectos positivos como la unión familiar, integración, confianza, comprensión, y armonía entre otros, pero sobre todo enfatizaron el apoyo, comunicación y afectividad en su hogar. Por el contrario afloran aspectos negativos como sobre protección (excesivo control de lo que hacen y mucha exigencia), discusiones, peleas por cosas insignificantes o relaciones hostiles. Según estos hallazgos hay de 30 a 40 por ciento de jóvenes en situación de riesgo. Al evaluar la comparación entre hombres y mujeres se conoció que las mujeres muestran mayor sensibilidad a los aspectos negativos que los hombres.

\section{Relación con padres, madres y encargados.}

La mayoría de jóvenes resaltan los aspectos positivos con sus padres madres o encargados, lo que se convierte en los primeros factores de protección para los jóvenes desde el ámbito familiar. Un promedio de 25 de cada 100 jóvenes no tienen o tienen muy poco apoyo esperado de sus padres.

\begin{tabular}{|c|c|c|c|c|}
\hline \multirow{2}{*}{$\begin{array}{l}\text { Cuadro 1. ¿Porqué no } \\
\text { vives con ...? }\end{array}$} & \multicolumn{2}{|c|}{ tu mamá? } & \multicolumn{2}{|c|}{ tu papa? } \\
\hline & Frecuencia & Porcentaje & Frecuencla & Porcentaje \\
\hline Porque murís & 11 & 10.5 & 36 & 20.2 \\
\hline Me abandonó & 5 & 4.8 & 8 & 4.5 \\
\hline Esta fuera del pais & 33 & 31.4 & 47 & 26.4 \\
\hline Tiene otra familia & 4 & 3.8 & 36 & 20.2 \\
\hline Trabaja en otra ciudad & 2 & 19 & 5 & 2.8 \\
\hline $\begin{array}{l}\text { Yo trabajo o estucio en } \\
\text { otra cludad }\end{array}$ & 16 & 152 & 11 & 6.2 \\
\hline Me casé & 26 & 24.8 & 18 & 10.1 \\
\hline Otra fazón & 8 & 76 & 17 & 9.6 \\
\hline Total & 105 & 100 & 178 & 1000 \\
\hline
\end{tabular}

Un promedio de 40 por ciento no vive con ninguno de sus padres. La mayor razón por la que no viven con su mamá o papá es porque está fuera del país, pero para los papás también sobresalen las razones de porque murió o tiene otra familia. Una vez más se evidencia que la emigración relacionada con la necesidad económica es la mayor causa desintegración familiar, así como la irresponsabilidad de progenitores como se muestra en las respuestas: "Me abandonó" o "porque tiene otra familia". Más de la mitad de éstos dejaron de vivir con uno de sus padres antes de los 5 años de vida y generalmente nadie tomó el lugar, aunque mencionan a otros familiares $\mathrm{y}$ otras personas.

Factores sociales. Se indagó la frecuencia con que los jóvenes participan en actividades periódicamente y lo que nunca hacen. Semanalmente la mayoría disfruta estar en casa y hacer tareas de la universidad; un poco más de la mitad va a la iglesia, de éstos, solo 3 de cada 10 participan en grupos religiosos. En porcentajes menores a 40 asisten semanalmente, cada mes o cada ciclo a lugares potenciales de riesgo como juegos de billar o electrónicos, discotecas y cine. Porcentajes menores a 20 nunca van a la iglesia; un porcentaje menor a 5 nunca hacen tareas de la universidad. Un poco más de la mitad nunca participa en grupos sociales ni religiosos, ni asisten a actividades culturales. Con lo anterior podría explicar el distanciamiento de la juventud actual con las realidades sociales.

Factores del contexto universitario. La mayoría percibe actitudes y comportamientos positivos como confianza, comprensión y aceptación en diferentes niveles entre sus mismos compañeros; sólo en porcentajes menores a 5 los perciben negativos en extremo. En porcentajes de 60 a 70 observan timidez y comportamiento inapropiado como escaparse de clases o simplemente los observan irresponsables; consideran que 'algunos' de sus compañeros son inconfiables y de tener cuidado. La mayoría (promedio $75 \%$ ) consideran que sus compañeros no consumen drogas ilícitas; sin embargo, al menos 2 de cada 10 saben de 'algunos' de sus compañeros que sí lo hacen, incluso son sus amigos; lo que hace pensar que existen pequeños grupos de universitarios que están consumiendo este tipo de sustancias y que estarían necesitando alguna orientación y formación de valores para evitar el consumo de drogas; se incluye la necesidad de ayudar especialmente a aquellos pequeños grupos que podrían estar necesitando rehabilitación. 
En relación al rendimiento académico, al menos 7 de cada 10 anotaron que "todos' y 'casi todos' pasan las asignaturas. El $16.2 \%$ percibe que ninguno deja asignaturas, 74.1 dice que 'algunos' dejan asignaturas; el $6 \%$ dice que 'todos' y 'casi todos' dejan asignaturas cada ciclo. Nuevamente se observa la tendencia de grupos menores a $30 \%$ en situación de riesgo.

Respecto a la percepción de agresividad como un factor potencial de riesgo, se distribuyen por mitad quienes consideran que 'ninguno' de sus compañeros es agresivo y los que consideran que 'algunos', el $6.5 \%$ consideran que 'todos' y 'casi todos' son agresivos.

Fn relación con docentes se indagó sobre fomento del individualismo, preferencia por parte de los docentes, fomento de confianza y amistad, consideración de sus problemas y necesidades como personas y no sólo como estudiantes. De 50 a 75 por ciento consideran positivos estos aspectos, en promedio de 25 por ciento los consideran negativos. Según los estudiantes, al menos el 20 por ciento de docentes saben de estudiantes que consumen drogas. creen que alrededor del 40 por ciento de docentes 'algunas veces' o 'nunca' sabe. Lo anterior indica la existencia de grupos de riesgo cuando estos aspectos no son favorables para todos.

Para indagar un poco sobre la influencia del modelaje se les preguntó a los estudiantes si conocían de conflictos entre los docentes, a lo que 6 de cada 10 contestó que 'nunca', un 25 por ciento 'algunas veces' y un 8.4 por ciento dice que 'casi siempre' y 'siempre' conocen de conflictos entre los docentes.

Casi la mitad llega a considerar que 'algunas veces' a sus docentes solo les interesa desarrollar el programa de estudios y no su persona. Ia mayoría $(80 \%)$ de estudiantes opina que sus docentes les motivan al análisis y discusión para afianzar sus conocimientos, el resto considera que 'alsunas veces' a 'nunca' lo hacen.

Al indagar sobre la satisfacción respecto al trato de la unwersidad, se encuentra una relación general de $75 / 25$ por ciento de satisfacción e insatisfacción respectioamente.

Se logró percibir que generalmente la Universidad no discrimina y que por el contrario presenta una visión integradora, ya que solo en porcentajes menores a 20 se opina lo contranio. Aunque, al indagar detalles al menos 6 de cada 10 percibe que la universidad promueve actividades sociales o comunales. Aunque, 4 de cada 10 que 'nunca' participan, y sólo un 21 por ciento dice que participa 'siempre' o 'casi siempre'. Se mantienc la tendencia porque la mayoría percibe positwamente las situaciones planteadas y cantidades menores a 40 por ciento las perciben como negativas. Lo que puede interpretarse como necesidad de volver estas situaciones positivas para que se constiluyan en factores de proteción.

Según los estudiantes lo más importante es tomar conciencia de su propia vida, que valoren la importancia de ellos mismos, "que hay que estudiar para triunfar, no para echar a la borda su vida" (mujer, Universidad Francisco Gavidia); aprender a diferenciar lo que les hace bien o mal; ocuparse en actividades productivas para su desarrollo, deportes, grupos culturales, grupos de apoyo social y comunal; saber elegir y discriminar a sus amigos "que sepan valorar el tipo de amistades que ellos tienen" (hombre, Unizersidad Católica de Oriente), "saber que un amigo que ofrece esto (droga) no es un amigo" (mujer, Universidad Tecnológica); buscar buenos consejos de sus padres, familiares y profesores para contar con ellos cuando tengan alguna dificultad y fortalecer la confianza con ellos para hablar cuando tengan problemas; leer más a cerca de los daños de las drogas para que nadie los engañe y fortalecerse con cl estudio de valores; participar en conferencias, charlas y programas de prevención para ayudarse y saber como ayudar a otros.

\section{A manera de conclusión}

Según el análisis de indicadores estudiados se advierte que alrededor de una tercera parte de población, sc encuentra en inminente riesgo: inseguridad individual. familiar y socioeconómica entre otras. La publicidad induce a la adopción de conductas inapropiadas y a consumos de drogas lícitas e ilicitas y de todo tipo de vicios. Si a ello se le suma, que la universidad prioriza la instrucción profesional y muy poco la educación integral; se genera una relación inversamente proporcional de factores de riesgo y protección: mientras se propicia el desgaste de factores de protección como la práctica de valores entre otros, se permite el aumento de factores de riesgo de todo tipo y con ello el consumo de sustancias psicoactivas. Ante el creciente riesgo, se advierte que la respuesta de la Universidad, familia y Estado debe ser inmediata para fortalecer los conocimientos científicos de causas y consecuencias de adicciones y fortalecer al autocontrol y autoestima entre los jóvenes.

\section{Recomendaciones}

Las recomendaciones se elaboraron a partir de los aportes de los mismos estudiantes y del análisis combinado de los argumentos teóricos de autores e informantes claves especialistas del problema en estudio.

\section{A las Universidades:}

La primera y más importante es que la Universidad tome conciencia de la proliferación del fenómeno de consumo de drogas, involucrando a todos sus docentes en tareas preventivas, enfatizando la importancia de la consideración de cada alumno como persona y no solo como sujeto de aprendizaje. Para poder hacer efectivo lo anterior es necesario:

Desarrollar permanentemente programas integrales de prevención durante el ciclo, que incluyan charlas de desarrollo de valores y campañas de prevención que expliquen los daños y consecuencias del uso de drogas. De manera que los estudiantes vayan tomando conciencia y apropiándose de los conocimientos necesarios para fortalecer su autocontrol y autoestima. 
Que los docentes introduzcan temáticas relacionadas durante cl desarrollo de sus cátedras y que procuren siempre la formación integral de los estudiantes, promover confianza y respeto en ambientes de aprendizaje armoniosos; equilibrar la exigencia sin represión o autoritarismo, promover relaciones horizontales docente-estudiante y no verticales.

Impulsar actividades culturales, deportivas y sociales cada ciclo, incorporando a grupos de estudiantes, de manera que éstos tengan más alternativas de sano esparcimiento y crecimiento cultural y social.

Dar mayor cobertura con los programas de orientación estudiantil, consejería y fuertes conocimientos científicos sobre las causas y consecuencias de la adicción; procurar relaciones más estrechas y de confianza para conocer sus perspectivas o formas de pensar y de esta manera saber en que momento tienen desequilibrios en su vida.

Aplicar proceso de detección de estudiantes con problemas de drogas, por medio de las instancias relacionadas, esto puede incluir el chequeo médico y pruebas extraídas en el laboratorio (sangre y orina) al ingreso de cada ciclo o al inicio de ciertas actividades; de manera que al detectar estudiantes con problemas, les puedan dar atención integral y confidencial para rehabilitarlos.

Aplicar reglamentación institucional (sin represión) equiparando con la concientización de los usuarios, esto puede incluir normas de 'no fumar' dentro de las aulas e instalaciones universitarias.

Celebrar el día internacional de "No fumar" en las Universidades que es el 31 de mayo de cada año paralelamente a una campaña de sensibilización de los daños.

\section{A las familias:}

Considerando que la familia es la base de la sociedad (Constitución de la República de El Salvador 1994), es momento de que los padres o responsables de familia retomen su papel como tales e inculquen en sus hijos de todas las edades, una comunicación asertiva, valores morales, espirituales y evitar por todos los medios posibles la desintegración familiar que tanto daño hace a la niñez y juventud actualmente. Aumentar los niveles de comunicación y confianza familiar.

\section{Al Estado:}

Establecer nexos con instituciones relacionadas a programas de prevención para unir esfuerzos y que dichos programas se desarrollen a nivel nacional y por otro lado establecer controles de la publicidad que induce al consumo, establecer controles efectivos para evitar la disponibilidad y tráfico de drogas.

Identificar a nivel poblacional niños y jóvenes considerados en riesgo por ausencia de sus padres y desarrollar programas de prevención atendièndoles adecuadamente.

\section{Fuentes de Información Consultadas}

1- Abelardo Diaz-Flores, DME Coordinador de Forlatecimiento Institucional, Investigación y Comunicación Social Comision Nacional Antidrogas. diciembre de 2006. III Encuentro de Iberoamericano de Observatorios Nacionales de Drogas. Cartagena de Indias, 12 de diciembre de 2006 Primer Esludio Nacional Sabre Consumo de Drogas en Población General de El Salvador. Documento en $p$ pl.

2- Becoña Iglesias Elisardo. Bases Cientificas de la Prevención de las Drogodefiendencias. Universidad de Santiago de Compostela. Delegación del Gobierno para el Plan Nacional sobre Drogas. Madrid, 2002. Documento en PDF:

3- Becoña Iglesias Elisardo. Bases Teónicas que sustentan los Programas de Prezención de Drogas. Unizersidad de Santiago de Compostela. Plan Nacional sobre Drogas. Madrid s/f. Documento en P'DF:

4- Comisión Interamericana para el Control y Abuso de Drogas (2000), CICAD-OEA. Evaluación del progreso de control de drogas 2001-2002

5. Comisión Satuadoreña Antudrogas (COSA-2005) Plan Nacional Antidrogas 2002 - 2008 (pp). El Salvador, Controamérica consultado en linea el $06 / 08 / 06$

6- Comisión Nacional Antidrogas 2006. Aplicación del curricule segundo paso en una escuela paroularia del departamento de San Salvador. Informe Ejecutivo. Fundación Antidrogas de El Salvador FUNDASALVA (1999). Perfil de pacientes atendidos ( $p p$ ). en linea consultado en linea el 06/08/06. hitp://www. fundasalva.org.sv/home/lsemestres05.pdf

7. Diario Oficial del 7 de noviembre de 2003. Decreto 153 de la Asamblea legislativa de la República de El Saluador.

8- Fundación Antidrogas de El Salvador FUNDASALVA (s/f) Comunicado de Prensa (pp 2-3). Consultado en linea el 06/08/06. wrow:fundasalia.org.se

9- Israet Rivas. Factores que incidieron en el consumo de drogas licitas e ilicitas. Fistudio realizado en instituciones de rehabilitación en el año 2006. Facultad de Giencias Sociales, Unizersidad Ezangélica de El Saluador.

10- Ley Reguladora de las actividades relativas a las Drogas (2003). Asamblea legislativa de la República de El Salvador, decreto 153, publicada en Diario Oficial el 7 de noviembre de 2003 , puesta en vigencia ese mismo año. Consultado en linea el 06/08/06

11-Organización de Estados Americanos (200.5). Lineamientos Hemisfericos de la CICAD en prevención escolar en formato PDF (pp. 12-22). Washington D.C. consultado en linea el 15/08/06.

12- Organización Mundial de la Salud (OMS). Obsenzatorios Sobm Drogas El Salvador 2007. Consultado el 29/06/07 en http? / / wewe.seguridad.gob.su/observatorio/drogas_ini, himl

13- Bhb Programa de las Naciones Limidas para el Desarrollo PNUD (2005). ¿Cuánto cuesta la violencia en El Salzador? $2^{\circ}$ ediciôn San Saluador. 